\title{
Application of a Configurable Model for Risk Assessment in the Work Sites
}

\author{
Fernando Oliveira Nunes \\ Instituto Superior de Engenharia de Lisboa (ISEL), Lisbon, Portugal \\ Email:fnunes@deea.isel.pt
}

How to cite this paper: Nunes, F.O. (2016) Application of a Configurable Model for Risk Assessment in the Work Sites. Open Journal of Safety Science and Technology, 6, 99-125.

http://dx.doi.org/10.4236/ojsst.2016.64009

Received: September 28, 2016

Accepted: December 12, 2016

Published: December 19, 2016

Copyright $\odot 2016$ by author and Scientific Research Publishing Inc. This work is licensed under the Creative Commons Attribution International License (CC BY 4.0). http://creativecommons.org/licenses/by/4.0/

\begin{abstract}
This paper presents a configurable model for occupational risk analysis, supported in decision matrix risk assessment with variable indexes dimensions and weightings. The formulation proposed seeks to overcome typical limitations such as range compression effect, mistakenly over classification of smaller risks and subjective input information. Also it allows its customization for each type of risk as a function of input information available in order to minimize subjectivity, while normalizing output for the calculated risk levels with a percent scale. This approach facilitates the implementation of models built to go along with the life of installations with periodically updates to fulfill accumulated data. To avoid discontinuation of procedures and practices implemented in the organizations, the model also enables the easy incorporation of risk assessment and control procedures based on risk classification matrixes, already in use regardless of their size and sector of activity.
\end{abstract}

\section{Keywords}

Configurable Model, Occupational Risk Assessment, Decision Matrix Risk Assessment, Multidimensional Risk Matrices, Severity Indexes

\section{Introduction}

According to the general principles established by law the employer is obliged to ensure their employees conditions of safety, hygiene and health in all aspects related to work, integrating all the activities in the enterprise, establishment or service and at all levels to assess risks to health and safety of workers, with the adoption of appropriate measures of prevention and protection, including, among others, reducing the risk of accidents and limiting exposures to physical, chemical and biological agents at work.

Occupational risk management is now required by the laws of safety, hygiene and health at work, in general, and specifically in some sectors of activity thus the lack of 
risk assessments carried out by the employer, may be grounds for disclaimer of insurers to pay claims due to accidents at work and occupational diseases. However, the legislation does not always accurately determine the criteria and benchmarks that must be taken into account in the assessment.

The process of hazard identification, risk assessment and risk control (risk management), must be dynamic, since it has to monitor all changes in activities that involve changes in processes, equipment and materials used. This dynamic process aimed at estimating the level of risks to safety and health of workers at work, resulting from circumstances in which the danger may occur in the workplace, aims to obtain the information necessary for the employer to meet conditions for taking appropriate decision about the type of control measures to be adopted.

In determining the control measures or changes to existing ones, to reduce risks, they must be established in accordance with the following hierarchy (general principles of prevention):

1) Hazard elimination or risk reduction through design;

2) Hazard replacement by less-hazardous methods or materials;

3) Engineered safeguards;

4) Warning and alerting techniques;

5) Administrative controls (e.g., safe work procedures, training);

6) Personal Protective Equipment (PPE).

The results of hazard identification, assessment and prioritization of risks and determination of necessary controls must be documented and kept current.

The residual risk remaining after the adoption of necessary control measures, is considered acceptable when it has been reduced to a level that can be tolerated by the organization to meet its legal obligations or others, if more stringent, resulting from policy safety and health for themselves assumed.

Risk management was initially introduced by large companies with the aim of reducing the cost of the insurance payment and at the same time, increase the protection of assets and employees.

The increased risk of industrial accidents from the use of more advanced and complex technologies, more raw materials, new processes and products, large capacity storage and transport of dangerous goods, increased the pressure on companies to reduce their risk, clarify the public about them and to adopt effective measures, emergency and risk containment. Moreover, with the evolution of society, issues related to ecological area began to worry the public and authorities. As a result, industries were forced to examine more accurately the effects of their internal and external operations.

In this sense, risk management emerges as a tool for reducing and managing the risks present in the industrial plants and offering technical support aimed at optimizing the use of technology, which undergoes an accelerated advance, and often inconsistent with the minimum safety standards that must be present in industrial activities. Risk management within an enterprise is the ability to assign safety and assurance to processes and procedures, components of your operating environment, allowing also the integra- 
tion of occupational and property safety.

It is fundamental, so that the risk assessment maintains a systematic approach, that the results obtained by implementing the measures proposed can be continuously evaluated and compared with alternative solutions.

To obtain the information necessary for this process one needs a formulation of the term "risk" that can be based on a model as complete as possible and making use of processing the largest number of variables available. This approach cannot however lead to the use of concepts too complex, subjective and out of context.

Risk management as a process of reducing the risks to a level deemed acceptable by society and to assure control, monitoring, and public communication [1], should then be based on the identification, analysis, evaluation and treatment of pure risks within a company, in order to minimize the possibility of occurrence of incidents and accidents, improving safety, reducing spendings on insurance and preserving the public image.

Although it can be implemented with the use of spreadsheets [2] [3] the proposed method built on a methodology of risk assessment matrices can also be part of information tools for managing Occupational Safety and Health [4].

\section{Risk Assessment in Occupational Context}

Occupational Risk is legally defined as the probability of realizing the damage depending on the conditions of use, exposition or interaction of the material component of the work that presents a danger. Said risk can also be defined as the combination of the likelihood of an occurrence of a hazardous event or exposure(s) and the severity of injury or ill health that can be caused by the event or exposure(s) [5].

In addition to the fulfillment of legal obligations, to quantify risks for a given work situation can be useful and necessary for certain situations:

- Getting to know the source of a problem and finding the most suitable control solution;

- To prove the cause-effect relationships in future development of professional diseases;

- Comparison with other work situation for use in epidemiological studies;

- Development, by researchers, of recommendations to be used in organizations.

The procedure for risk assessment should take into account all routine and occasional activities, considering all the people with access to workplaces. It should also include all infrastructure, equipment and existing materials in the workplace and take into account human factors such as behavior and abilities. As such, the number of items greatly increases with the dimension of the organizations.

Many of the methods available for risk analysis had their genesis associated to the more hazardous activities like, chemical and petrochemical, nuclear, military, etc., with approach characteristics often too independent from the variables associated with the work context (environment work, worker, executed tasks, etc.). Examples are HAZOP (Hazard and operability Study) [6] and FMEA (Failure Modes and Effects Analysis) [7], among many others [8] [9]. 
Despite the many existing definitions and different origins, whose compilation can be found on the website of Human Factors and Ergonomics Society (www.hfes.org), human factors can be understood as a set of knowledge about the capabilities, limitations and other human characteristics. The availability of that information is used ultimately for the design of tools, machines, systems, tasks, functions, and environment for a safe, comfortable and effective human use [10], i.e., human engineering or human factors.

However, the capabilities, limitations and other human characteristics, physical or mental, as well as being inputs to the human engineering, are also naturally indispensable factors in any estimation of the risk present in the human activities related to work.

In fact, one of the types of risks where more evaluation methods have been developed specifically aimed at work situations, is thus of musculoskeletal injuries related to work. Indeed one can list a significant number of currently available methods for assessing those types of risks (non-exhaustive list): the OWAS method (Ovako Working Postures-Analysis System) [11]; the RULA method (Rapid Upper Limb Assessment) developed at the University of Nottingham's [12]; the Kilbom method (Guidelines for "practitioners") [13]; the SI method (Strain Index) [14]; the HAL method (Hand Activity Level) which had its origin in the range of Latko et al. [15]; the REBA method (Rapid Entire Body Assessment) [16]; the OCRA method (Occupational Repetitive Actions) [17]; the LUBA method (Loading on the Upper Body Assessment) [18] and; the MAPO method (Movement and Assistance of Hospital Patients) [19].

The above listed methods are, in greater or lesser extent, difficult to implement since they require expertise to characterize the activity from the working point of view for the various factors used for each: duration of the task, the total daily duration, strength applied, frequency of action, repeatability, speed of movement, muscular work, as well as the existence of breaks or other activities.

In the context of psychosocial and organizational risk factors Cox et al. [20] report that in addition to the work context the content of the work may be associated with several risk factors related to psychosocial activity. Thus, the execution of repetitive or monotonous tasks, the lack of operating space, the lack of opportunity to learn and high quantitative or qualitative requirements are examples of those risk factors.

To overcome the difficulties in applying several specific methods to assess multiple different risks, other methodologies have been developed that allow quantification (estimate) of the risk associated with the hazards identified more generically in the workplace.

Such methods search to build a risk assessment matrix (or risk classification matrix), which is a method to display the combinations of probability and severity and to categorize those combinations, became very popular among safety professionals to communicate with and influence decision makers.

One of the oldest and best known is the method of W. Fine [21] that by proposing scales to consider the consequences, the exposure factor and a probability factor, assesses the risk through the product of those three factors. This method also introduces 
the concept of assessing the case for investing in corrective measures, by calculating an index of justification, which measures the degree of hazard in the risk assessment obtained by a factor of cost of investment, and also by the degree of correction achieved. This approach thus seeks to address the economic component and the expected result, in the prioritization of control measures to be implemented.

In the same vein the method of Kinney and Wiruth [22], using very similar scales also calculates risk with an analogous concept (degree of risk presented graphically), using three equivalent factors: the probability, the exposure and the possible consequences.

Numerous variations for those methods have been developed within individual projects and organizations, creating their own or tailoring existing risk matrixes, seeking to meet the felt needs of the original methods unreliability given the information available. In fact, many standard decision matrixes risk assessment can be found today in different risk contexts [23] [24] [25] [26] [27].

One specific example at safety context is the method known as Simplified System for Accident Risk Assessment (SSARA) [28], which calculates the level of risk by the product of a level of probability by a level of consequences. The probability level is in turn calculated as the product of a deficiency level by an exposure level. For the three factors are given appropriate tables.

Above methods essentially seek to provide tools with which to establish a basis for comparing risks of different nature. For example, a fractured arm in a fall from height, getting a back pain when lifting a load, intoxication when handling a specific chemical, professional deafness contract after a certain number of years, etc., are, in principle, incomparable risks, since they have their origin in risk factors that are characterized by parameters of different nature. Some of these parameters are well defined in the applicable legislation and standards, but others may only be specified by the criteria of common sense.

As pointed out by Cox [29], decision matrix risk assessment methods have been widely praised and adopted as simple, effective approaches to risk management. They provide:

- clear framework for systematic review of individual risks and portfolios of risks;

- convenient documentation to rationalize risk rankings and priority setting;

- relatively simple appearing inputs and outputs, often with attractively colored grids;

- opportunities for many stakeholders to participate in customizing category definitions and action levels; and

- opportunities for consultants to train different parts of organizations on "risk culture" concepts at different levels of detail, from simply positioning different hazards within a predefined matrix;

- no special expertise is needed in quantitative risk assessment methods or data analysis.

\section{Mathematical Modelling of Occupational Risk}

In short, methods for professional risks assessment will always consist of mathematical 
models, somewhat validated, containing more or less factors (parameters or variables) according to the complexity that is accepted to reach, always bearing in mind a committed relationship between this complexity with all its differentiation and interdependence, and the usability of the developed methods.

\subsection{Classical Model Definition}

BS 8800 [30] and BS OHSAS 18,001 [5] specify that the calculation of risk must take into account the likelihood and potential severity of harm resulting from exposure to the existing hazard. In other words, there is now consensual agreement that risk assessment shall include:

- the probability of occurrence of a lesion or damage to health;

- the maximal foreseeable severity of such lesion or damage to health.

Thus, the assessment of occupational hazards arising from an activity must take into account the likelihood of exposure to each of the hazards present and the severity of the consequences of these exposures. Like the probability of occurrence, as a probabilistic variable, the representation of severity may also be performed numerically between 0 and 1 , so the risk can be quantified by:

$$
R=P \times S
$$

where:

$$
\begin{aligned}
& R \text {-Risk }(>0 \text { and } \leq 1) ; \\
& P \text {-Probability }(>0 \text { and } \leq 1) ; \\
& S \text {-Severity }(>0 \text { and } \leq 1) .
\end{aligned}
$$

According to this definition, the risk varies in direct proportion to its probability of occurrence and the severity of its consequences. Figure 1 illustrates two curves of constant risk levels: A and B, where risk levels B > risk levels A. Same risk level can arise from two different situations, as for example: a fall from a scaffold (low probability $P$ with high severity $S$-point $\mathrm{A}_{1}$ ) and a fall at the same level in slippery floor (high probability $P$ with low severity $S$-point $\mathrm{A}_{2}$ ).

Despite the complexity that may arise in risk assessments of certain situations, the representation of constant risk curves allows a simple and useful illustration for the

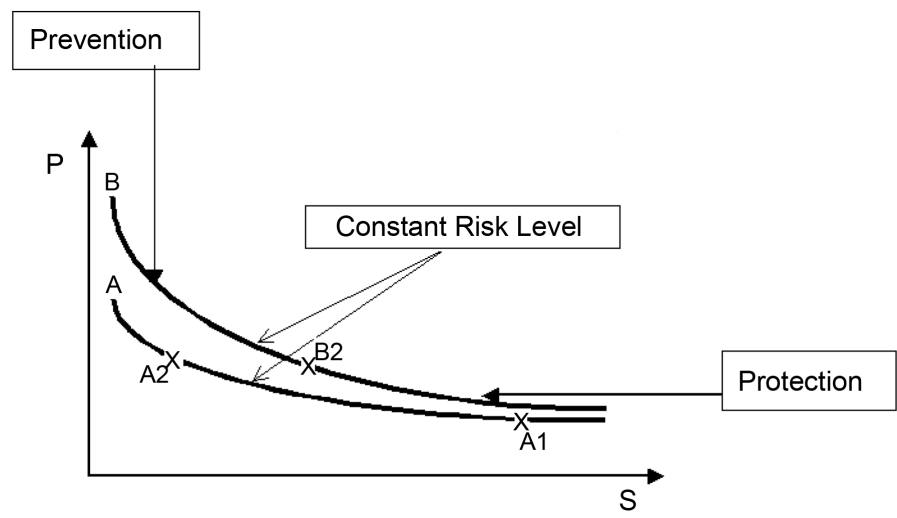

Figure 1. Curves of constant risk levels. 
effects of the implementation of control measures (prevention and protection) on the resulting level of risk:

- Preventive measures are mainly aimed at reducing the probability (possibly canceling) of occurrence of exposure;

- Protection measures, collective or individual, seek to reduce the consequences during an exposure (to reduce and eventually annul the severity of damage and injury caused by exposure).

Alternatively, to the formulation of "risk" while probabilistic variable, represented by Equation (1), has been used the concept of "risk level". This concept can also be achieved using probability and severity factors graduated in integer scales from 1 to $N$, instead of real values from 0 to 1 , so as to be called, Probability Index ( $P$ scored between 1 and $N_{P}$ ) and Severity Index ( $S$ scored between 1 and $N_{S}$ ).

Most methods based on decision matrixes risk assessment estimate risk levels (or equivalent concept) by the product of the factors chosen for the particular method. Consequently, output values obtained for the risk levels vary strongly with the scales chosen for those factors, making it harder to interpret intended outputs.

Accordingly to Pasman et al. (p. 769) [31]: "Intuitive and qualitative methods can do much, but plant complexity may be large and communication on risk may become difficult without formal methodology. Quantitative risk analysis offers much, but has its weaknesses and drawbacks. The required effort is considerable, specialists are needed, and variability in answers is large. Yet, a model built to go along with the life of an installation and updated periodically may be very useful".

The use of configurable models based on decision matrix risk assessment allows for customization of the procedure for identifying hazards and assessing and controlling risks for each particular organization, and allows the individualization of the analysis for each type of risk. This individualization also allows a better updating of relevant information through its connection to the management of Occupational Health and Safety.

However, can be argued [29] [32] that existing risk assessment matrices experience several difficulties arising from their fundamental mathematical and logical limitations making it harder to assess risks:

1) Can correctly and unambiguously compare only a small fraction (e.g., less than $10 \%$ ) of randomly selected pairs of hazards. They can assign identical ratings to quantitatively very different risks ("range compression"), due to geometric effect of Equation (2);

2) Can mistakenly assign higher qualitative ratings to quantitatively smaller risks. For risks with negatively correlated frequencies and severities, they can be useless, leading to random decisions;

3) Categorizations of severity cannot be made objectively for uncertain consequences. Inputs to risk assessment matrices (e.g., frequency and severity categorizations) and resulting outputs (i.e., risk ratings) require subjective interpretation, and different users may obtain opposite ratings of the same quantitative risks. 
Malchaire \& Koob [33] also experienced many subjectivities in the Kinney \& Wiruth method [22] in assessing risks at a given workplace when carried out by observers with different formation and experience.

\subsection{New Approach to Decision Matrix Risk Assessment}

Risk level $(R L)$ can be obtained from Equation (2), with normalized values in a percent scale (range between 1 and 100), which has the advantage of being independent of the number of levels $N_{P}$ and $N_{\mathcal{S}}$ enabling a free adoption of more adequate scale ranges for the selected indexes of a particular model based on decision matrix risk assessment.

$$
R L=\frac{P \times S}{N_{P} \times N_{S}} \times 99+1
$$

where:

$$
\begin{aligned}
& R L \text {-Risk Level (1\% to } 100 \%) ; \\
& P \text {-Probability Index (1 to } N P \text { ); } \\
& S \text {-Severity Index (1 to NS); } \\
& N_{P}-\text { Maximum value of } P \text { index; } \\
& N_{S} \text {-Maximum value of } S \text { index. }
\end{aligned}
$$

The normalized scale of results (in percent) avoids the difficulties of interpretation of multiple risk ratings that result from the numerous methods of decision matrix risk assessment that have been developed.

Figure 2 illustrates the map of values obtained for the risk levels with the use of Probability and Severity Indexes in the same scales, 1 to 15. It can be seen that due to the mathematical model used, the values are too geometrically scaled, i.e., only the first (short) values of the range of possible outcomes (1\% to 100\%) occupies the entire bottom left of the matrix leading that the highest risk levels are represented fewer times in the top right of the matrix ("range compression" effect).

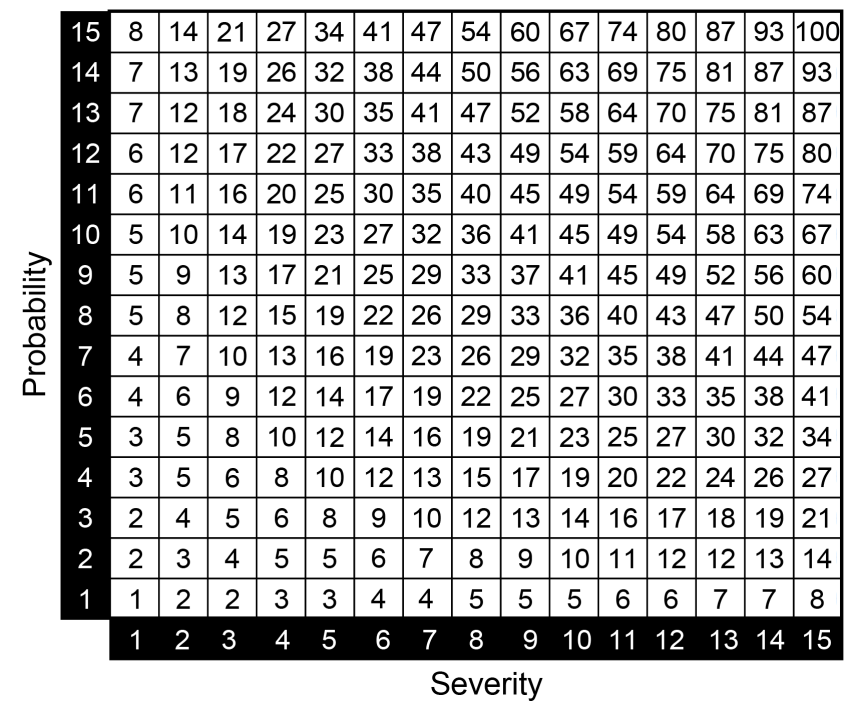

Figure 2. Risk levels in a multiplicative model. 
To obtain a better distribution of the values calculated for the risk levels, one can eliminate the geometric effect previously reported applying log function to the products of Equation (2), maintaining at same time a percent scale. The Risk Level is then to be calculated by:

$$
R L=\frac{\log (P)+\log (S)}{\log \left(N_{P}\right)+\left(N_{S}\right)} \times 99+1
$$

where the meaning of the variables is the same as Equation (2).

To show the effect of last formulation, Figure 3 illustrates the risk levels obtained for the risk levels with the use of Probability and Severity Indexes also at the same scales.

It may be noted that the values obtained are better distributed and more evenly fill the array, avoiding pointed difficulty \#1 in section 3.1. One can also see a more linear dependence between the indexes and the calculated risk levels: if one of the indexes is at the beginning of the scale and the other at the end, i.e. one with the minimum value (1) and the other with the maximum value (15), then the resulting risk level calculated with Equation (3) is in the middle of its range, i.e., 51\% (50.5\% rounded to integer). A better distribution of risk levels, avoiding assign identical ratings to quantitatively very different risks, can also be seen in Figure 3 a much better discrimination of the risks associated with probabilities and severities with weak correlations, as opposed to the distribution observed in the multiplicative model and partially overcoming difficulty \#2 pointed out in section 3.1 .

Risk is relative to the observer. It is a subjective thing and depends upon who is looking. Some writers refer to this fact by using the concept "perceived risk" [34]. Also, risk depends on what you do, what you know and what you do not know (uncertainty).

When trying to quantify risk, we must begin by the distinction between probability and frequency. Probability as we use it is a numerical measure of a state of knowledge, a degree of belief or a state of confidence. Frequency refers to the outcome of an experiment of some kind involving repeated trials, at least conceptually then, frequency is a

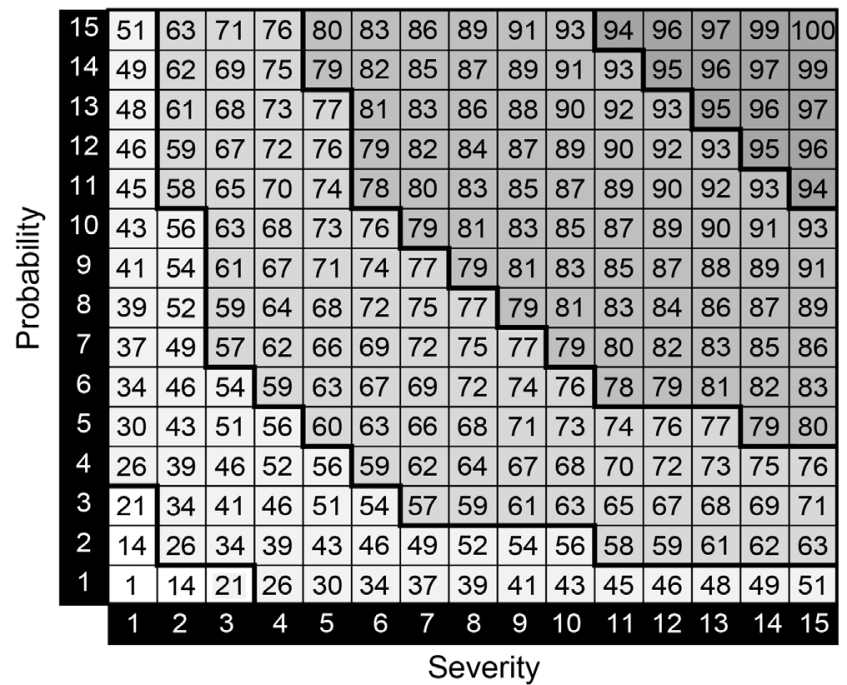

Figure 3. Risk levels in a log model. 
well-defined, objective, measurable number. If one has insufficient data, there is nothing else that can be done but using probability [34].

So, when we don't have statistical data (frequency) one can try to estimate probability using the information available as approaching other concepts like "exposition" to existing hazards in the workplace, "deficiency" of the safety conditions associated with the identified hazard and the effective "safety culture" within the organization.

Generalizing Equation (3) to accommodate a total number of $K$ indexes we can maintain a normalized approach to percent output for the calculated risk levels.

$$
R L=\frac{\sum_{i=i}^{K} \log \left(F_{i}\right)}{\sum_{1=i}^{K} \log \left(N_{i}\right)} \times 99+1
$$

where:

$R L$-Risk Level (1\% to $100 \%)$;

$F_{i}-I^{\text {th }}$ index ( $i=1$ to $\left.K\right)$;

$N_{i}$ - Maximum value for $F_{i}$ index.

To reduce pointed subjectivity of the input information (Difficulty \#3 of section 3.1) one must use as many derivate concepts of probability as possible. Of course their relative weight in the calculation of the risk level of each particular situation must be differentiated. With the proposed log formulation, differentiation can be achieved, without unjustified extensions and discontinuities in the scales of the indexes used, with weighting factors applied to each index entered in the calculation of risk levels. This is not possible with the formulation of Equation (2) where the indexes cannot be individualized.

Consequently, one can improve model configurability through the addition of weighting factors to the indexes of Equation (4) in order to provide a way to differentiate their contributions to the calculation of the risk levels. Normalized Equation (5) also maintains percent output of the calculated risk levels.

$$
R L=\frac{\sum_{i=i}^{K} W_{i} \log \left(F_{i}\right)}{\sum_{1=i}^{K} W_{i} \log \left(N_{i}\right)} \times 99+1
$$

where:

$R L$-Risk Level (1\% to $100 \%)$;

$F_{i}-i^{\text {th }}$ index $(i=1$ to $K)$;

$W_{i}$-Weighting factor for $i^{\text {th }}$ index $(i=1$ to $K)$;

$N_{i}$ - Maximum value of index $F_{i}$.

In fact, this last formula for the calculation of risk levels allows customization of risk assessment matrices as regards:

- Variable number of indexes used in calculating the risk level (number of inputs);

- Variable scale (maximum value) and importance (weighting factor) of each index in the calculation of risk level;

- Invariable scale (percent) for the calculated values for the risk levels (outputs). 
The direct dependence of different weightings for each index can be seen in Figure 4, for an example with only two indexes, that were assigned the importance of twice for Probability index relatively to the Severity index, i.e., $W_{P}=2 W_{s}$. It can be noted that when $S$ takes the maximum value (15) but $P$ takes the minimum value (1), the risk level reaches only $1 / 3$ the maximum scale value (34\%). At the other end of the array, by changing values between the two indexes, i.e., $S=1$ and $P=15$, then the level of risk reaches $2 / 3$ the maximum scale value (67\%), translating the double influence of $P$ over $S$. As a result of this mathematical formulation, it is thus a close proportionality of the weight of each index in the calculated risk levels.

\subsection{Deficiency Index on Occupational Risk Assessment}

The severity or the likelihood effectively involved in the achieved level of risk, are conditioned by all kinds of risk control measures, i.e., safety barriers, functions and systems [35] [36] [37] [38] that are effectively implemented. These conditions can be translated by using a Deficiency index with adequate weighting to the reliability of the information of the occupational health and safety (OHS) management system. For the specification of the Deficiency index several sources of information can be used:

- Results of studies conducted on the duration and frequency of tasks performed, as well as the conditions under which they are developed;

- Training actually received by the workers involved usually and occasionally and others who may be affected;

- Facilities, equipment and hand tools that can be used;

- Distance that materials must be moved manually, size, shape, surface and weight of these materials;

- Substances used and displayed on the job, physical state of the substances used or exposed (liquid, smoke, dust);

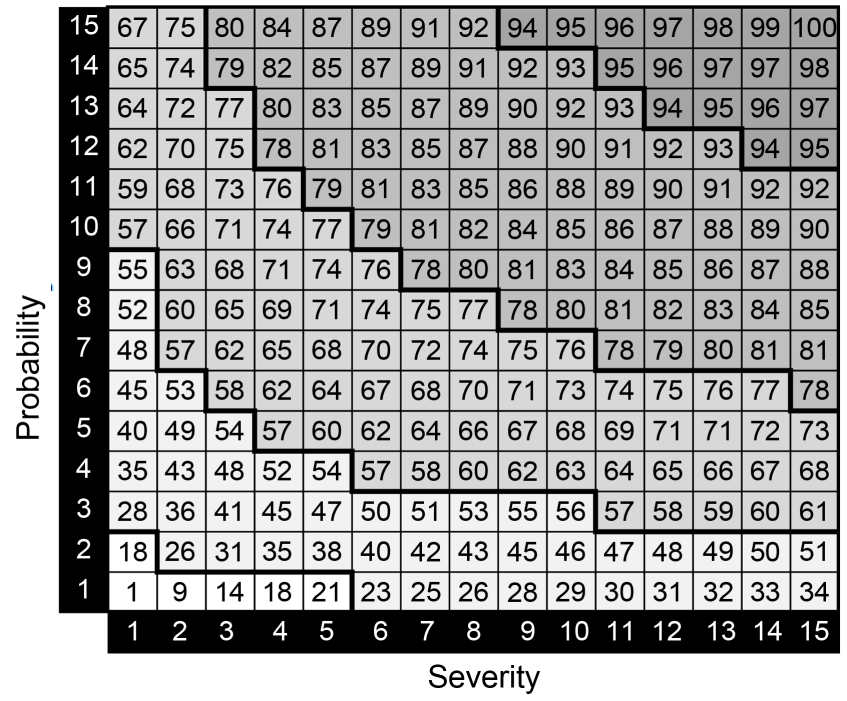

Figure 4. Influence of weighting the indexes in the calculation of $R L\left(W_{P}=2 W_{S}\right)$. 
- Adequate signalization (demarcations, labels, warnings, emergency);

- Existence of procedures for authorization of work, manufacturer's instructions for operation and maintenance of facilities, equipment and tools, ancillary services, content and appropriate recommendations on safety data sheets of substances used or exposed;

- Actual knowledge of legal requirements, regulations and relevant regulatory related with work, facilities, equipment and tools used, control devices and protection supposedly running;

- Results of safety inspections and audits conducted in the workplaces.

\subsection{Severity Index Improvement}

To provide useful information that can be used as a satisfactory overall measurement for the severity of the exposition to hazards in the workplace, overcoming difficulty \#3 pointed out in section 3.1, one can consider several approaches.

Cuny \& Lejeune [39] [40] use number of days off work to establish precise levels of the severity scale combining estimations of a dangerous event probability and probabilistic distribution of severity levels for the consequences of this event.

Gennarelli \& Wodzin [41] pointed out several dimensions of severity, including threat to life, mortality, amount of energy dissipated/absorbed, hospitalization need, treatment cost, treatment complexity, length of treatment, temporary and permanent disability, permanent impairment and quality of life, but without establishing a measurement scale.

Using functional block diagrams (FBD) as logical models in the quantification of occupational risks, Papazoglou \& Ale [42] decompose "undesirable health consequences" into "dose" and "dose/response"; "dose" is decomposed to "center event" and "mitigation"; "center event" is decomposed to "initiating event" and "prevention". This specific FBDs together with their equivalent event trees are used to delineate the various accident sequences that might lead to "injury or death consequences".

Aneziris et al. [43] also using risk logic models for six types of falls from a height (ladders, scaffolds, roofs and roof openings, holes in the ground, moveable platforms and non-moving vehicles) propose scales to assess levels of consequence severity such as recoverable injuries, permanent injuries and lethal injuries.

An approach to develop severity functions for some accident modes [44] at construction sites may consider predictors related to the amount of energy dissipated and/or absorbed that can be evaluated in situ such as heights, speeds, weights, and morphology of moving vehicles. One can also use the biomechanical limits of the human body as determined in several studies.

In fact, the most practical approach that can be made in the management of occupational health and safety is to use legal or regulatory frameworks that somehow rate the level of expected consequences for existing hazards in the workplace. In the absence of those references logical criteria should be using for classification of severity based on amounts of harmful energy released and/or absorbed that can be expected during the exposure to danger, compared with the limits supported by humans without risk to 
their health and safety.

For instance, based on criteria established in Directives 2006/121/EC, 18th December, and 2008/112/EC, 16th December, of the European Parliament and of the Council, one can compose a classification for severity in a particular task according to existing information of products used. In Material Safety Data Sheets (MSDS) available information is indicated as threshold limit values (TLV), time-weighted average (TWA), short duration (STEL) or maximum concentration (Ceiling) to chemicals in humans. It is also possible to establish relationships between the lethal dose $\left(\mathrm{LD}_{50}\right)$ and/or lethal concentration $\left(\mathrm{LC}_{50}\right)$, with the aforementioned values of MSDS, thus matching the rate of toxicity (Table 1 ).

In some cases, legal information can even provide direct criteria, as pointed out in Table 2 on the severity index for occupational noise exposition (2003/10/CE, 6th

Table 1. Possible criteria for establishing severity indexes for the products used in a particular task.

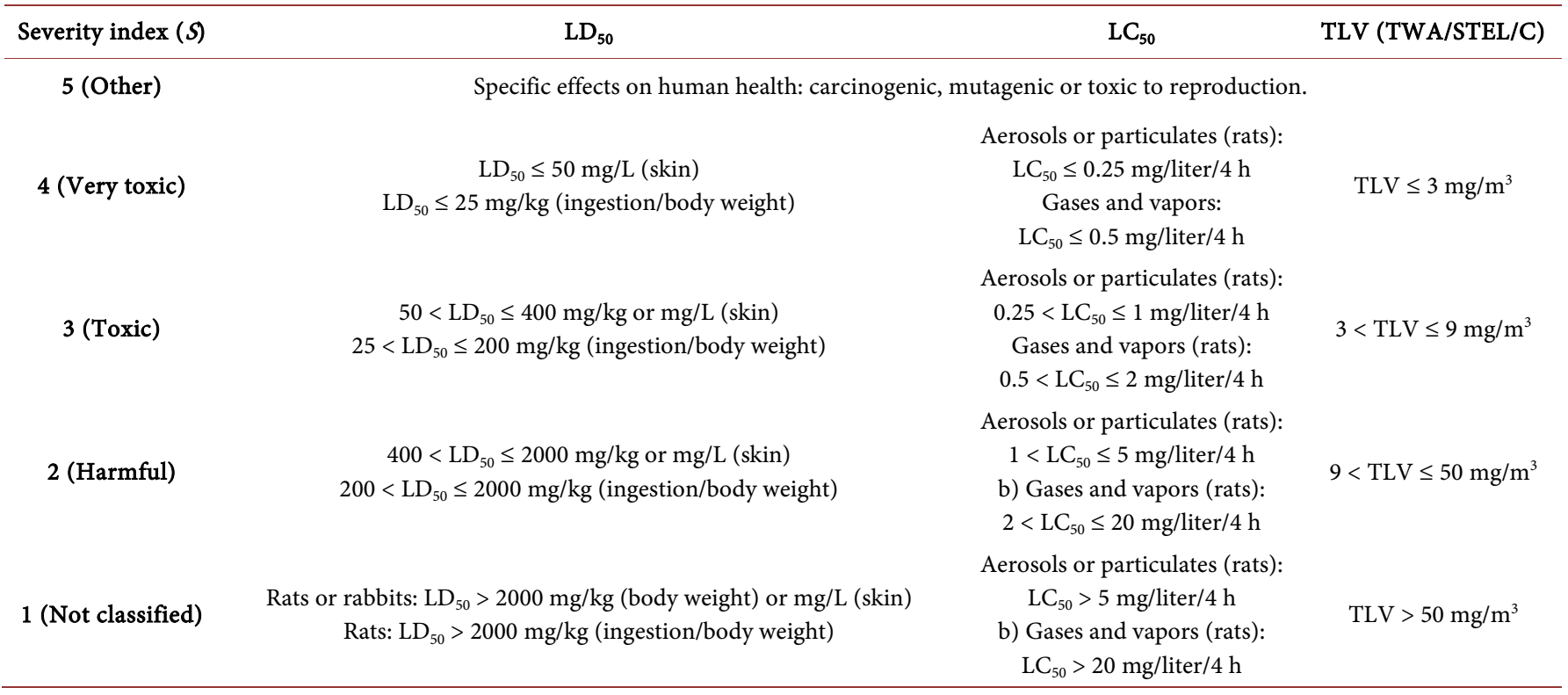

Table 2. Severity indexes for occupational noise exposition.

\begin{tabular}{cc}
\hline Severity index $(S)$ & Noise measured \\
\hline 5 & $\mathrm{~L}_{\mathrm{EX}, 8 \mathrm{~h}}>87 \mathrm{~dB}(A)$ \\
4 & $\mathrm{~L}_{\mathrm{Cpeak}}>140 \mathrm{~dB}(C)$ \\
& $85<\mathrm{L}_{\mathrm{EX}, 8 \mathrm{~h}} \leq 87 \mathrm{~dB}(A)$ \\
$\mathbf{3}$ & $137<\mathrm{L}_{\mathrm{Cpeak}} \leq 140 \mathrm{~dB}(C)$ \\
& $80<\mathrm{L}_{\mathrm{EX}, 8 \mathrm{~h}} \leq 85 \mathrm{~dB}(A)$ \\
$\mathbf{1}$ & $135<\mathrm{L}_{\mathrm{Cpeak}} \leq 137 \mathrm{~dB}(C)$ \\
& $\mathrm{L}_{\mathrm{EX}, 8 \mathrm{~h}} \leq 80 \mathrm{~dB}(A)$ \\
& $\mathrm{L}_{\text {Cpeak }} \leq 135 \mathrm{~dB}(C)$ \\
\hline
\end{tabular}


February, of the European Parliament and of the Council). In addition to the use of weighting factors of Equation (5), the severity scale can be used to limit the expected consequences for each type of exposure to risk. In this case severity index 5 is unclassified, because expected consequences shall not achieve dead or equivalent severity as specified later (Table 6).

Research undertaken to obtain information from selected free-fall impacts [45] determines that resulting severity is determined by the age and sex of the victim, location and height of the fall, body orientation at impact, body impact position and type of surface impact.

Cory \& Jones [46] simulations for assessing the impact response, and potential to cause injury, of domestic floor surfaces during short falls shows the potential to discriminate between differences in surface mixture. Findings suggest that the injury potential of impact surfaces are influenced by factors such: material properties of the top surface layer and the thickness of underlay (if present) comprising the upper surface mixture; material properties of the underlying surface (wood, chipboard and concrete); and structure of the underlying surface (joints cross supports or between joints and supports).

Most work situations can easily provide data for:

- height of fall (determines velocity at surface impact);

- type of surface impact (elasticity of the surface which determines force exerted on the fallen body) and presence of dangerous structures.

Following Aneziris et al. [43] dose approach one can use Severity indexes from Table 3 when assessing risk for free-fall accidents that uses more than five indexes $\left(N_{S}=6\right)$.

For AC current contacts IEC 60479-1:2005 "Effects of current on human beings and livestock" refers the value of $40 \mathrm{~mA}$, for a current path from left hand to feet, as the threshold to $5 \%$ probability of heart fibrillation, which is not a unique value but rather varies with duration of contact. The value increases with short duration contacts and varies according to the current path through the human body. For instance, with the same probability (5\%) and for the same current path (left hand to feet), the value of 40 $\mathrm{mA}$ increases to $90 \mathrm{~mA}$ for contact durations above $5 \mathrm{~s}$. Also, the impedance of the human body varies inversely with the applied voltage, with contact area and with environment: dry conditions, water-wet conditions and saltwater-wet conditions.

Table 3. Severity indexes for free-fall accidents.

\begin{tabular}{ccc}
\hline Severity index $(\mathcal{S})$ & Height of fall $[\mathrm{m}]$ & Type of surface \\
\hline 6 & $\mathrm{H}>10$ & Hard/Dangerous structures \\
5 & $2<\mathrm{H} \leq 10$ & Hard/Dangerous structures \\
4 & $\mathrm{H}>10$ & Soft \\
3 & $\mathrm{H} \leq 2$ & Hard/Dangerous structures \\
2 & $2<\mathrm{H} \leq 10$ & Soft \\
1 & $\mathrm{H} \leq 2$ & Soft \\
\hline
\end{tabular}


Protection of persons in electrical installations against indirect contact (situations when a person touches a metal conductive part that has been accidentally energized by insulation fault on a device or an electrical machine) is based on combining measures affecting both the characteristics of the equipment (insulation class 0, I, II or III) and the automatic disconnection of the power supply using medium sensitivity devices (30 $\mathrm{mA}<\mathrm{I} \Delta_{\mathrm{n}} \leq 500 \mathrm{~mA}$ ) combined with earthing of the equipment, to eliminate those situations quickly, before anyone comes into contact with the metal conductive part or, the use of PELV (Protection by Extra Low Voltage). Non-dangerous voltage is $50 \mathrm{~V}$ (conventional limit value) but lower supply voltage values of $25 \mathrm{~V}$ or $12 \mathrm{~V}$ are used for operating conditions in wet or submerged environments, respectively.

On power socket circuits, in damp rooms, mobile installations (building sites, fairs, etc.), agricultural buildings or when the earthing conditions are defective or unknown, people must be protected by residual current operating devices, which operate at $30 \mathrm{~mA}$ or less (high sensitivity devices).

IEC/TR 60479-5:2007 “Touch voltage threshold values for physiological effects" provides touch voltage-duration combination thresholds based on analysis of information concerning body impedances and current thresholds of physiological effects, as given in IEC/TS 60479-1. Such threshold combinations relate to specific environmental and contact conditions that determine body impedance for particular current pathways. So, risk assessment must include voltage threshold values (taking into account contact area, skin moisture condition, body current pathway), which is not practicable in most working conditions.

To approach predictable touch voltage-duration in practical situations one can use Severity indexes from Table 4 when assessing risk for activities with electrical equipment $\left(N_{S}=11\right)$.

Configurability of the model can easily accommodate different scales for the Severity

Table 4. Severity indexes for electrical contacts.

\begin{tabular}{ccc}
\hline Severity index $(S)$ & Protection measures & Environment \\
\hline 11 & Defective or unknown protection & Submerged \\
10 & Medium sensitivity device with earthing & Submerged \\
9 & Defective or unknown protection & Wet \\
$\mathbf{8}$ & Defective or unknown protection & Dry \\
$\mathbf{7}$ & Medium sensitivity device with earthing & Wet \\
$\mathbf{6}$ & High sensitivity device & Submerged \\
$\mathbf{5}$ & High sensitivity device & Wet \\
$\mathbf{4}$ & Medium sensitivity device with earthing & Dry \\
$\mathbf{3}$ & PELV & Submerged \\
$\mathbf{2}$ & PELV & Wet \\
$\mathbf{1}$ & High sensitivity device & Dry \\
\hline
\end{tabular}


indexes associated to the different kinds of risk with the adjustment of the respective maximum value $\left(N_{S}\right)$ on Equation (5).

\section{Application to a Generic Model}

In order to demonstrate the use of configurable model presented in the form of a generic model based on decision matrix risk assessment, a predefined model for its application in occupational risks assessment is established.

Predefined model of Equation (5) is presented with five indexes (see section 4.1 for further explanation), thus supported by the following equation:

$$
R L=\frac{W_{F} \log (F)+W_{E} \log (E)+W_{S} \log (S)+W_{D} \log (D)+W_{C} \log (C)}{W_{F} \log \left(N_{F}\right)+W_{E} \log \left(N_{E}\right)+W_{S} \log \left(N_{S}\right)+W_{D} \log \left(N_{D}\right)+W_{C} \log \left(N_{C}\right)} \times 99+1
$$

where:

$R L$-Calculated risk level (1\% to $100 \%)$;

$F$-Frequency index ( 1 to $\left.N_{F}\right)$;

$E$-Exposition index (1 to $N_{E}$ );

$S$-Severity index (1 to $\left.N_{S}\right)$;

$D$-Deficiency index (1 to $\left.N_{D}\right)$;

$C$-OHS Culture index (1 to $N_{C}$ );

$W_{\Gamma}$-Weighting factor for the index $I$ (with $I=F, E, S, D$ and $C$ );

$N_{I}$-Maximum value for the index $I$ (with $I=F, E, S, D$ and $C$ ).

Scales with five classes, often used in association with checklists (Likert scales [47]), are very popular for the classification of the indexes. So, using all the indexes on scales from 1 to 5 , and making $W_{F}+W_{E}+W_{S}+W_{D}+W_{T}=100$, Equation (6) can be simplified to the form:

$$
R L=1.416\left\lfloor W_{F} \log (F)+W_{E} \log (E)+W_{S} \log (S)+W_{D} \log (D)+W_{T} \log (C)\right\rfloor+1
$$

Calculated Risk Levels with 4 indexes equally weighted and scaled from 1 to 5 are shown in Figure 5. Again one can see the same behavior already described in section 3.2. Now, if two of the indexes are at the beginning of the scale and the other two at the end, i.e. two with the minimum value (1) and the other two with the maximum value (5), then the resulting risk level calculated with Equation (6), with only 4 indexes, is in the middle of its range, i.e., $51 \%$.

\subsection{Indexes Specification for the Generic Model}

For the specification of the Frequency index $-F$ (Table 5) may be considered available data on "frequency rates" and "incidence rates" of new cases of occupational injury [48], as well as the memory of workers and technicians involved in the analyzed activities.

In a still young system of risk assessment model can be used with a limited number of indexes. In the absence of reliable data, allowing the specification of the Frequency index, or in the complete absence, a rate of exposure may be used instead to fulfill an Exposition index. Exposition index-E (Table 5) results from percent counts of the 

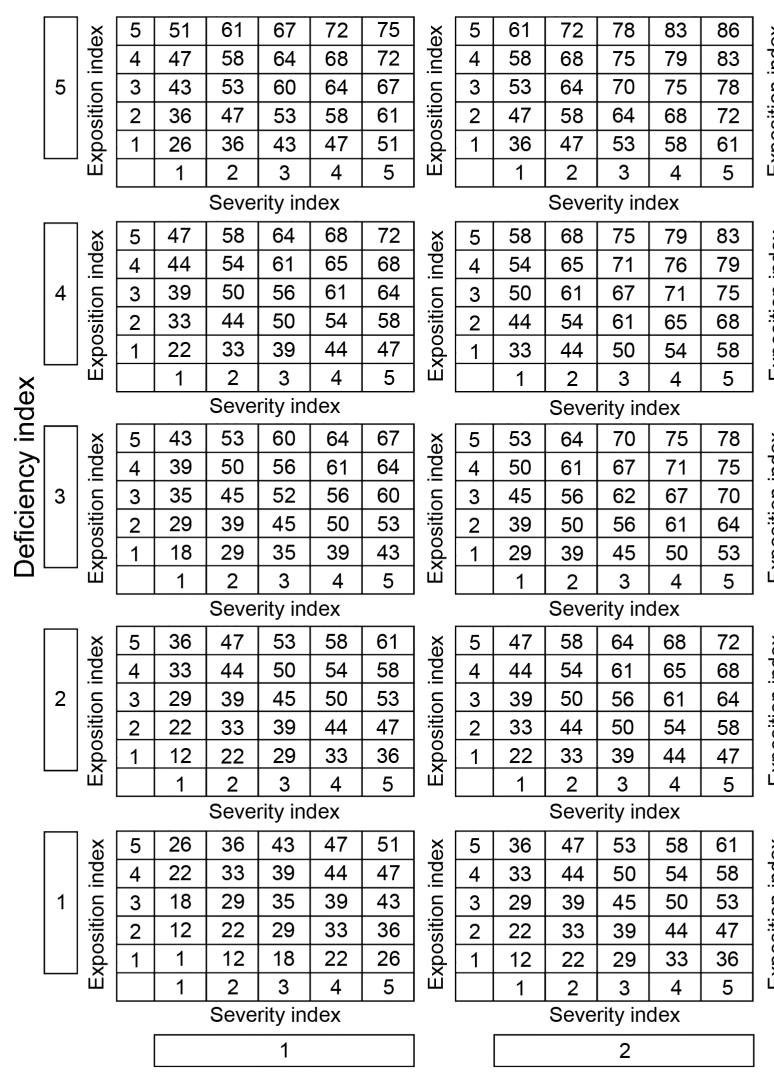

Severity index

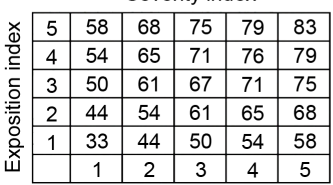

Severity index

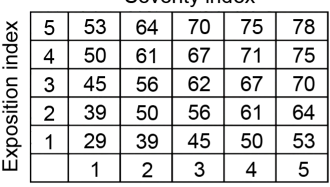

Severity index

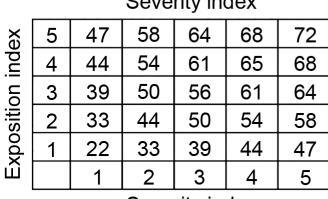

Severity index

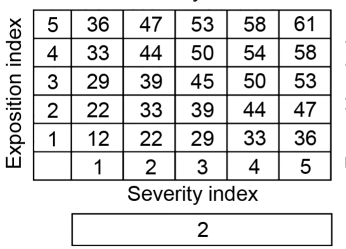

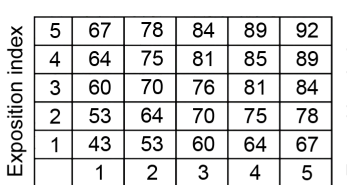

Severity index

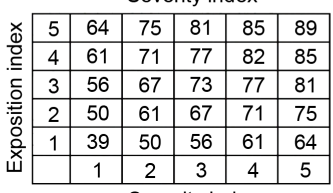

Severity index

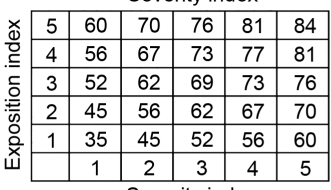

Severity index

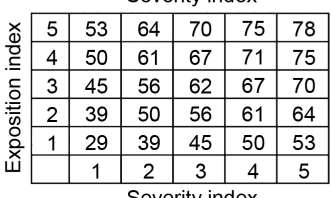

Severity index

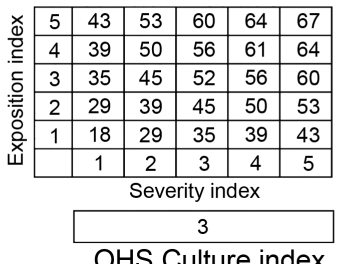

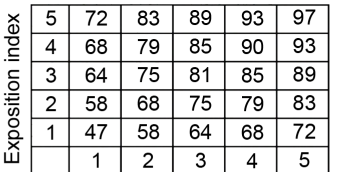

Severity index

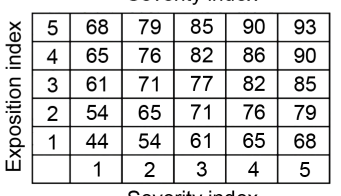

Severity index

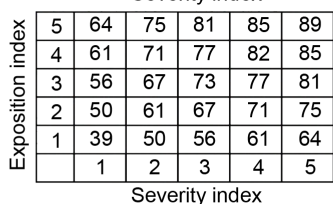
Severity index
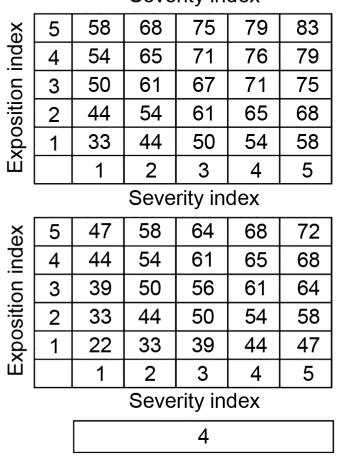

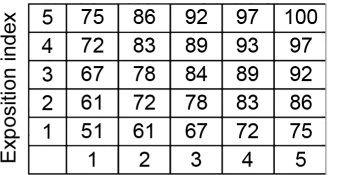

Severity index

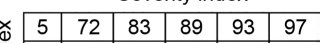

\begin{tabular}{|l|l|l|l|l|l|}
\hline 5 & 72 & 83 & 89 & 93 & 97 \\
\hline 4 & 68 & 79 & 85 & 90 & 93 \\
\hline
\end{tabular}

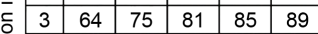

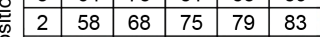

\begin{tabular}{|c|c|c|c|c|c|c|}
\hline \multirow{2}{*}{$\dot{0}$} & 1 & 47 & 58 & 64 & 68 & 72 \\
\cline { 2 - 6 } & & 1 & 2 & 3 & 4 & 5 \\
\hline
\end{tabular} Severity index

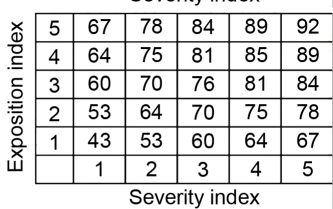

Severity index

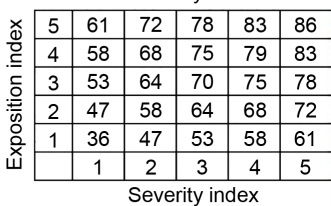

Severity index

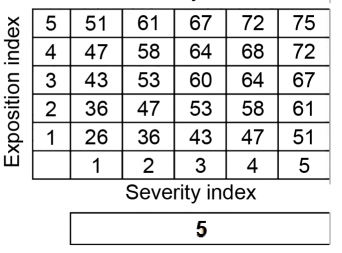

Figure 5. Risk levels in a log model with 4 indexes.

durations of exposures observed or planned.

General criteria for the specification of Severity index $-S$ are presented in Table 6. Available data on "severity rate" and "days lost" of new cases of occupational injury [48] may also be considered, as well as specifically defined criteria for the type of risk analyzed, as previously seen in Table 1, Table 2, Table 3, and Table 4.

Table 7 presents general criteria for the Deficiency index as defined in section 3.3. In a matured management system, along with the previous indexes, the use of Deficiency index can be supported by the results of audits and other indicators of performance from the OHS management system and specific studies on the activities and workplaces under evaluation.

Occupational health and safety culture (OHS culture), can be seen as a concept for exploring how informal organizational aspects influence OHS in a positive or negative way [49]. It can have an impact on how OHS is perceived and dealt with among workers in an organization, and on whether workers are aware of OHS-related issues and act in a safe and healthy way. Also referred as safety culture (safety-related beliefs, attitudes, and values that interact with an organization's systems, practices, people, and leadership to establish norms about how things are done in the organization) and safety climate (shared perceptions of safety policies and procedures by members of an organization at a given point in time, particularly regarding the adequacy of safety and consistency 
Table 5. Criteria classification for frequency index or exposition index.

\begin{tabular}{|c|c|c|}
\hline $\mathrm{F} / \mathrm{E}$ & Frequency & Exposition \\
\hline 5 & One or more times per day. Happens "all the time". & work time $>90 \%$ \\
\hline 3 & One or more times per month. Some people remember this type of event/situation. & $25 \%<$ work time $\leq 50 \%$ \\
\hline 2 & One or more times per year. Someone remembers this type of event/situation. & $5 \%<$ work time $\leq 25 \%$ \\
\hline
\end{tabular}

Table 6. Criteria classification for severity index.

\begin{tabular}{lr}
\hline S & Severity \\
\hline 5 & Possibility of death, injury or illness with permanent total disability or very severe. \\
4 & Injury or illness with temporary total disability, permanent partial or severe. \\
3 & Injury or illness with temporary partial disability, or moderately severe. \\
2 & Possibility of injury without disability or mild health problems. \\
1 & No injuries nor health problems. \\
\hline
\end{tabular}

Table 7. Criteria classification for deficiency index.

\begin{tabular}{lr}
\hline D & Deficiency \\
\hline 5 & Safety barriers, function and systems nonexistent or unknown. \\
4 & Serious deficiencies in existing ones or does not comply with legislation; Needs several improvements. \\
3 & $\begin{array}{r}\text { Absence and/or some deficiencies in existing ones, respecting part of the law, presents some problems in } \\
\text { situations of abnormal operation. }\end{array}$ \\
2 & $\begin{array}{r}\text { Sufficient ones but upgradeable, respecting the law, with very few requirements below the established } \\
\text { standards. }\end{array}$ \\
\hline 1 & Sufficient and well established ones, respecting international standards, above the required by law. \\
\hline
\end{tabular}

between actual conditions compared to espoused safety policies and procedures) [50], it's a complex concept that has been the subject of extensive research since the eighties. Nevertheless, existing tools for exploring and assessing the organization's safety culture/climate, can be used to specify OHS Culture indexes $(C)$ to be accounted for in Equation (7).

\subsection{Risk Ranking}

After quantification (regardless of the risk analysis methodology to be used, more or less quantitative) it must be applied to the assessment of risk according to a predefined range so as to inform the implementation of control measures.

This process of risk assessment is part of a process of risk management resulting from the hazard(s) identified, taking into account the adequacy of existing controls, the result is the decision of the acceptability or not of risk by the organization, taking into account their legal obligations and its own OHS policy [5]. 
Risk ranking is the final step of risk assessment and is a process through which one makes value judgments about the acceptability of risk, taking into account a number of factors and criteria. It is a process of comparing the value obtained in step risk analysis and a framework of acceptable risk.

With this comparison we can anticipate the need and type of intervention to achieve, since the mere act of monitoring, control action to bring the risk to acceptable levels, or even interrupt or interdict work where have been found a risk of a certain magnitude.

Following BS 8800 [30] approach Table 8 provides a set of relevant technical and emergency actions, depending on the value corresponding to the level of risk estimated. Risk rank 1 classifies the risk as acceptable, and risk rank 2 as tolerable and so only better cost-benefit solutions to control risks must be considered. Risk rank 3, 4 and 5 require growing specific actions and urgency to control the risk.

Limits for the risk rank ranges have been selected from the combinations of equal number of indexes with nested values, as can be seen in Figure 5, i.e., with equivalent combinations of the $K=4$ indexes as follows: $(1,1,2,2),(2,2,3,3),(3,3,4,4)$ and (4, $4,5,5)$ or; $(1,2,1,2),(2,3,2,3),(3,4,3,4)$ and $(4,5,4,5)$ or; $(2,2,1,1),(3,3,2,2),(4$, $4,3,3)$ and $(5,5,4,4)$ or; $(2,1,2,1),(3,2,3,2),(4,3,4,3)$ and $(5,4,5,4)$. The obtained limits are independent of the number of indexes $(K)$ of the model used.

In the case of a computerized OHS management system, the model can benefit from a semi-automatic configuration of the indexes used in calculating risk levels [51]. This configuration is accomplished via the information contained in accumulated records of incidents and accidents, results of audits and measurements of hazard parameters, level of protective equipment, training, information and consultation, and health surveillance.

Table 8. Ranking of the estimated risk level.

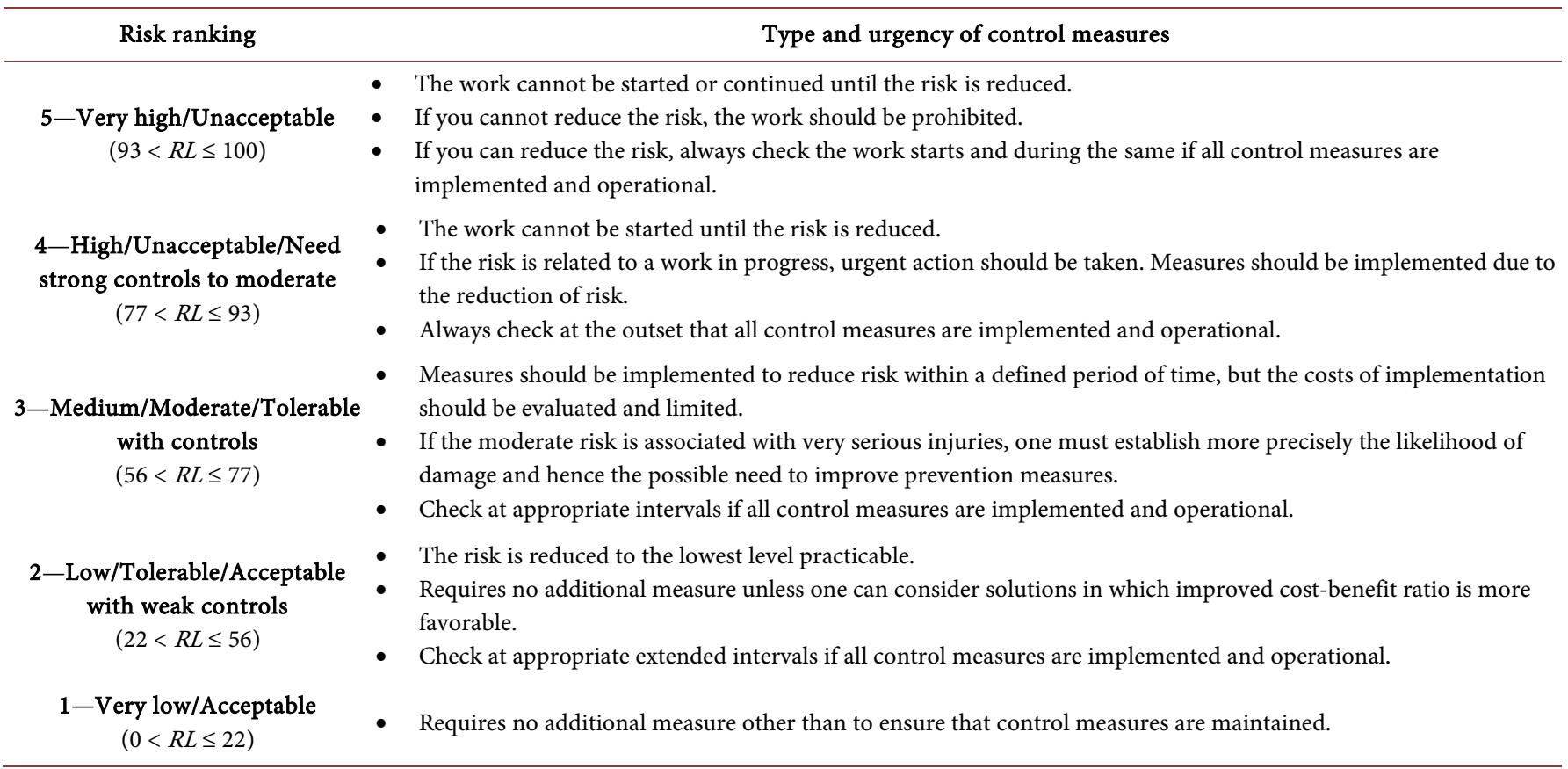


Still new risk assessment procedures can present some difficulties in their assembly, which relate, among others, failure history and records of incidents and accidents, and occupational diseases and work-related diseases.

In the case of a computerized OHS management system, the model can benefit from a semi-automatic configuration of the indexes used in calculating risk levels [51]. This configuration is accomplished via the information contained in accumulated records of incidents and accidents, results of audits and measurements of hazard parameters, level of protective equipment, training, information and consultation, and health surveillance.

Still new risk assessment procedures can present some difficulties in their assembly, which relate, among others, failure history and records of incidents and accidents, and occupational diseases and work-related diseases.

\subsection{Weighting Factors Estimation and Comparison with Other Decision Matrix Risk Assessment Methods}

As previously introduced the addition of weighting factors to the indexes provides a way to differentiate their contributions to the calculation of the risk levels. It's also the best way to configure the model for multiple occupational contexts.

Estimation of weighting factor for the indexes can be reduced to a typical problem of multiple linear regression with least square estimation, where we want to estimate the $\beta$ parameters of the dependent variable y with independent variables $x$.

$$
y=\beta_{0}+\beta_{1} x_{1}+\beta_{2} x_{2}+\beta_{3} x_{3}+\beta_{4} x_{4}+\beta_{5} x_{5}
$$

where, being $\beta_{0}$ equal to zero, one obtains the following relations:

$$
\begin{array}{lll}
y=R L-1 & & \\
x_{1}=1.416 \log (F) ; & \text { with } \quad \beta_{1}=W_{F} \\
x_{2}=1.416 \log (E) ; & \text { with } \quad \beta_{2}=W_{E} \\
x_{3}=1.416 \log (S) ; & \text { with } \quad \beta_{3}=W_{S} \\
x_{4}=1.416 \log (D) ; & \text { with } \quad \beta_{4}=W_{D} \\
x_{5}=1.416 \log (C) ; & \text { with } \quad \beta_{5}=W_{S}
\end{array}
$$

Let's take the example of six risk factors/hazards identified in a work front where a group of safety practitioners agreed among them in assigning the values indicated in Table 9 for the three indexes used: Table 5, Table 6, and Table 7, making $W_{F}=W_{T}=0$ in Equation (7); as well as the correspondent percent risk levels (in 5\% steps), to each observed risk factor. During the risk assessment the group freely consulted risk rank values of Table 8.

Table 10 shows estimated values for the weighting factors by applying multiple linear regression. Estimation has been achieved with an R square (coefficient of determination) value of $99.95 \%$ and an adjusted $\mathrm{R}$ square of $66.59 \%$, which was penalized by the relatively few samples (6) when compared with the number of independent variables (3).

Thus, in the absence of specific quantitative models and more precise data inputs, the 
corresponding "tuned" risk assessment model of Equation (7) for the observed risk factors as seen by the group of safety practitioners, can be expressed by:

$$
R L \%=1.416\lfloor 6.86 \log (E)+73.08 \log (S)+20.06 \log (D)\rfloor+1
$$

To validate the model let us make the comparison of the number of index levels and maximum level for the index $F_{p}$ in three decision matrix risk assessment methods widely used in the practice of the organizations, indicated in Table 11.

Applying log transformation to the universe of the combinations of the three indexes values of each method and normalizing to percent scale, the estimated values for the weighting factors by applying multiple linear regression, are shown in Table 12 . $R$ square values obtained show that the three methods are very closely represented by the applied formulation. Using the estimated weighting values and the required limits for the risk rank ranges of each method, they can be closely translated by the present

Table 9. Assigning made by the group of safety practitioners.

\begin{tabular}{ccccc}
\hline Work situation & $E$ & $S$ & $D$ & $R L \%$ \\
\hline $\mathbf{1}$ & 3 & 4 & 3 & 75 \\
$\mathbf{2}$ & 5 & 5 & 4 & 95 \\
$\mathbf{4}$ & 3 & 3 & 3 & 65 \\
$\mathbf{5}$ & 2 & 2 & 2 & 40 \\
$\mathbf{6}$ & 4 & 3 & 3 & 70 \\
\hline
\end{tabular}

Table 10. Estimated weighting factors.

\begin{tabular}{cc}
\hline Weighting factor & Estimated value \\
\hline$W_{E}$ & 6.86 \\
$W_{S}$ & 73.08 \\
$W_{D}$ & 20.06
\end{tabular}

Table 11. Methods comparison of the index $F_{i}$.

\begin{tabular}{cccc}
\hline Risk Assessment Method & $F_{1}$ & $F_{2}$ & $F_{3}$ \\
\hline W. Fine [21] & $P(6 / 10)$ & $C(6 / 100)$ & $E(6 / 10)$ \\
Kinney \& Wiruth [22] & $P(7 / 10)$ & $C(6 / 100)$ & $E(6 / 10)$ \\
SSARA [28] & $D(3 / 10)$ & $C(4 / 100)$ & $E(4 / 4)$ \\
\hline
\end{tabular}

Table 12. Estimated weighting factors for the methods.

\begin{tabular}{ccccc}
\hline Risk Assessment Method & $F_{1}$ & $F_{2}$ & $F_{3}$ & R square/Adjusted R square \\
\hline W. Fine & 19.60 & 19.25 & 19.32 & $99.993 \% / 99.523 \%$ \\
Kinney \& Wiruth & 20.20 & 19.76 & 19.83 & $99.987 \% / 99.585 \%$ \\
SSARA & 15.56 & 15.47 & 15.53 & $99.999 \% / 97.777 \%$ \\
\hline
\end{tabular}


configurable model, meaning that the influence of the discontinuous scales used for the indexes can be replaced by weighting factors while maintaining continuous integer scales, which can be more easily rationalized.

\section{Application to Particular Cases}

To demonstrate the application of the model to different types of hazards (with different severity scales) will be considered a front work in a trench where have been identified four risk factors/hazards with different establish criteria for the severity indexes $(S)$ as shown in Table 1, Table 2, Table 3 and Table 4:

1) Chemical classified as toxic to be used in parts cleaning for about $2 / 3$ of working time. Appropriate protective masks are available, which for some shorter handling operations are not used;

2) Noise emitted by a machine running during the entire working time and achieving sound pressure levels between 82 and $84 \mathrm{~dB}(A)$. Earplugs are available and workers were trained in their use;

3) Fall inside of the trench containing equipment and materials for the pipe assembly with a depth of three meters with exposure occurring for about $1 / 3$ of working time in which the worker is out of the ditch or getting in and out. There are access ladders but no guardrails;

4) Electrical equipment has to be used for about $1 / 3$ of working time even when the trench has water from rain or infiltrations. Electrical installation have automatic disconnection of the power supply using medium sensitivity devices $\left(30 \mathrm{~mA}<\mathrm{I} \Delta_{\mathrm{n}} \leq 500\right.$ $\mathrm{mA}$ ) combined with earthing of the equipment but are not inspected regularly.

Choosing exposure indexes $(E)$ from Table 5 and deficiency indexes $(D)$ from Table 7 , as well as the weighting factors estimated in section 4.3 (Table 10), the calculated risk levels of Equation (6) associated with the four risk factors identified are presented in Table 13.

Results in Table 13 can help to decide for the application of the following measures to control risk levels:

1) Risk Level \#1 can be reduced to $56 \%$ (rank 3 to rank 2) if use of protective masks is guaranteed in all handling operations $(D=1)$;

2) Risk Level \#2 can be reduced to $16 \%$ (rank 2 to rank 1 ) if noise level from machine can be reduced to less then $80 \mathrm{~dB}(A)(S=1)$;

3) Risk Level \#3 can be reduced to $63 \%$ (rank 4 to rank 3) if guardrails and bodyguards on the access ladders above 2 meters high are mounted $(S=3)$ and to $51 \%$ (rank

Table 13. Calculated risk levels in the work front.

\begin{tabular}{cccccc}
\hline Risk factor & $E$ & $S$ & $D$ & $R L \%$ & (Rank) \\
\hline 1) Use of toxic product (Table $\left.1: S=3 ; N_{S}=5\right)$ & 4 & 3 & 3 & 70 & (3) \\
2) Noise from machine (Table $\left.2: S=2 ; N_{S}=5\right)$ & 5 & 2 & 2 & 48 & $(2)$ \\
3) Fall to trench (Table 4: $\left.S=5 ; N_{S}=6\right)$ & 3 & 5 & 3 & 85 & $(4)$ \\
4) Electrical contact (Table 5: $\left.S=10 ; N_{S}=11\right)$ & 3 & 10 & 5 & 95 & (5) \\
\hline
\end{tabular}


3 to rank 2) if everyday inspections are performed $(D=1)$;

4) Risk Level \#4 can be reduced to $91 \%$ (rank 5 to rank 4) if regular inspections are performed to the electrical installations $(D=3)$ and to $74 \%$ (rank 4 to rank 3 ) if high sensitivity device are used $(S=6)$. If PELV is used $(S=3)$ one can reach $R L=51 \%$ (rank 3 to rank 2).

After application of above mentioned measures to control risks, one can see that the risk level \#1, \#3 and \#4 have been reduced to the lowest level practicable, requiring no additional measure unless one can consider solutions in which improved cost-benefit ratio is more favorable (rank 2). Risk level \#2 simply requires no additional measure other than to ensure that the controls are maintained (rank 2).

\section{Results and Discussion}

Intuitive and qualitative methods such as risk assessment matrices (decision matrix risk assessment) have been widely adopted as simple and effective approaches to risk management, because they provide clear framework for systematic review of individual risks and portfolios of risks with relatively simple appearing inputs and outputs (with optionally colored grids), convenient documentation for the rationale of risk rankings and priority setting, and require no special expertise in quantitative risk assessment methods or data analysis.

The configurability of the present method, achieved through the use of any number of indexes and the values assigned to weighting factors (from zero to cancel the contribution of the respective index in the model until another value, which determines the importance of the index in the estimation of risk), allows the development and improvement of the procedure for risk assessment as the mature state of the OHS management system of each organization.

Typical difficulties arising from current risk assessment matrices in assigning identical ratings to quantitatively very different risks, qualitative ratings to quantitatively smaller risks and poor categorizations of severity, require subjective interpretation and lead to the attainment of opposite ratings from different users. To overcome those difficulties, it was necessary to improve their fundamental mathematical and logical limitations to a better fit to real workplaces. This goal was achieved with the development of a configurable method for risk assessment based on decision matrix risk assessment that easily accommodate different scales to categorize severity arising from particular hazards, as well as for any other index used, without modifying the output percent scale. Weighting factors also allows the use of continuous integer scales for the indexes. Altogether, those features contribute to more clear and understandable models to risk management activities.

Indeed, decision matrix risk assessment based on a model built to go along with the life of an installation and updated periodically with accumulated data are very useful in a OHS managing system. However, one can't expect similar accuracy with uncertainty as other more complex methods of quantified risk analysis, based on objective classical statistics. 


\section{Conclusions and Further Work}

In addition to other more specific legal obligations, under general principles of prevention set out also in the general obligations of the employer (89/391/EEC, 12th June, of the Council), the activity of identification, analysis and control of hazards, deserves very special references, and is even profusely referred in the reference documents with requirements for the management of OHS.

In that sense the control measures implemented must be preceded and match the results of risk assessments associated with various stages of the production process, including preparatory activities, maintenance and repair.

Moreover, current required forms of the annual reports of activity of OHS services often determine a structure for reporting the hazards identified in all places and work processes, which greatly increase the number of items with the dimension of the organizations. The information demanded is based on a standard classification and coding of physical, chemical, biological, ergonomic, psychosocial and organizational, and other hazards, as well as the respective control measures adopted, urging the organization to adopt a more coherent OHS management system, aimed at the adoption of uniform classification of hazard and the respective control measures, thus establishing a common language between OHS services.

Risk classification matrices simplify occupational risk assessments by reducing the required inputs and calculations to a comprehensive set of judgments, while making the rating logic, transparent and easy to apply. These features have driven the spread of this methodology among safety practitioners.

Presented method for assembly customized risk classification matrices helps growing procedures for risk assessment in all stages of the OHS managing system, regardless their size and sector of activity. Nevertheless, it is necessary to develop further Severity indexes in the several work contexts, i.e., diverse types of risk, in order to minimize subjectivity arising from different users.

\section{References}

[1] Zimmerman, R. (1986) The Management of Risk. In: Covello, V.T., Menkes, J. and Munpower, J., Eds., Risk Evaluation and Management, Plenum, New York, 435-460. https://doi.org/10.1007/978-1-4613-2103-3_18

[2] Madeira, C.P. (2007) Matriz de quantificação do risco químico profissional em laboratório. In: Soares, C.G., et al., Eds., Riscos Públicos e Industriais, Edições Salamandra, Lisboa, 935-950.

[3] Graça, H., Batista, S. and Nunes, F.O. (2012) Risk Assessment in Analytical Laboratories. International Symposium on Occupational Safety and Hygiene, Occupational Safety and Hygiene-Proceedings SHO 2012, Guimarães, February, 288-293.

[4] Nunes, F.O. (2003) Avaliação de Níveis de Segurança nos Locais de Trabalho: Uma Abordagem Quantitativa [Assessment of Safety Levels at Workplace: A Quantitative Approach]. Proceedings of the 3 th International Colloquium on Occupational Safety and Hygiene, Portuguese Association of Engineers, Oporto, March, 77-82.

[5] BS OHSAS 18001 (2007) Occupational Health and Safety Management Systems: Require- 
ments. British Standard Institution.

[6] Lawley, H.G. (1974) Operability Studies and Hazard Analysis. Chemical Engineering Progress, 70, 105-116.

[7] US Army (1949) Procedure for Performing a Failure Mode Effect and Criticality Analysis. United States Military Procedure, MIL-P-1629.

[8] Tixier, J., Dusserre, G., Salvi, O. and Gaston, D. (2002) Review of 62 Risk Analysis Methodologies of Industrial Plants. Journal of Loss Prevention in the Process Industries, 15, 291303. https://doi.org/10.1016/S0950-4230(02)00008-6

[9] Oliveira, C.G. (2011) Proposal for an Integrated Approach to Professional Risk Assessment. $\mathrm{PhD}$ Thesis, Universidad de León, Leon.

[10] Chapanis, A. (1991) To Communicate the Human Factors Message, You Have to Know What the Message Is and How to Communicate It. Human Factors Society Bulletin, 34, 1-4.

[11] Karhu, O., Kansi, P. and Kuorinka, I. (1977) Correcting Working Postures in Industry: A Practical Method for Analysis. Applied Ergonomics, 8, 199-201.

https://doi.org/10.1016/0003-6870(77)90164-8

[12] McAtamney, L. and Corlett, N. (1993) RULA: A Survey Method for the Investigation of Work-Related Upper Limb Disorders. Applied Ergonomics, 24, 91-99. https://doi.org/10.1016/0003-6870(93)90080-S

[13] Kilbom, A. (1994) Repetitive Work of the Upper Limb Extremity: Part I-Guidelines for the Practioner. International Journal of Industrial Ergonomics, 14, 51-57. https://doi.org/10.1016/0169-8141(94)90005-1

[14] Moore, J.S. and Garg, A. (1995) The Strain Index: A Proposed Method to Analyze Jobs for Risk of Distal Upper Extremity Disorders. American Industrial Hygiene Association, 56, 443-458. https://doi.org/10.1080/15428119591016863

[15] Latko, W.A., Armstrong, T.J., Foulke, J.A., Herrin, G.D., Ranbourn, R.A., and Ulin, S.S. (1997) Development and Evaluation of an Observational Method for Assessing Repetition in Hand Tasks. American Industrial Hygiene Association, 58, 278-285. https://doi.org/10.1080/15428119791012793

[16] Hignett, S. and McAtamney, L. (2000) Rapid Entire Body Assessment (REBA). Applied Ergonomics, 31, 201-205. https://doi.org/10.1016/S0003-6870(99)00039-3

[17] Occhipinti, E. and Colombini, D. (2005) The Occupational Repetitive Action (OCRA) Methods: OCRA Index and OCRA Checklist. Handbook of Human factors and Ergonomics Method, CRC Press, Boca Raton.

[18] Kee, D. and Karwoswki, W. (2006) An Assessment Technique for Postural Loading on the Upper Body (LUBA). The Occupational Ergonomics Hand Book: Fundamental and Assessment for Occupational Ergonomics. CRC Press, Boca Raton, 832-839.

[19] Battevi, N., Menoni, O., Ricci, G.M. and Cairoli, S. (2006) MAPO Index for Risk Assessment of Patient Manual Handling in Hospital Wards: A Validation Study. Ergonomics, 49, 671-687. https://doi.org/10.1080/00140130600581041

[20] Cox, T., Griffiths, A. and Rial-Gonzalez, E. (2000) Work-Related Stress. Office for Official Publications of the European Communities, Luxembourg.

[21] Fine, W.T. (1971) Mathematical Evaluations for Controlling Hazards. Journal of Safety Research, 3, 157-166.

[22] Kinney, G.F. and Wiruth, A.D. (1976) Practical Risk Analysis for Safety Management. Technical Publication 5865, Naval Weapons Center, California.

[23] GAO (1998) Combating Terrorism: Threat and Risk Assessments Can Help Prioritize and 
Target Program Investments. National Security and International Affairs Division, United States General Accounting Office.

[24] ICAO (2013). Safety Management Manual (SMM). Document 9859, International Civil Aviation Organization.

[25] ISO 17666 (2003) Space Systems Risk Management. International Standards Organization.

[26] NASA (2009) Risk Management Reporting. GSFC-STD-0002, Goddard Space Flight Center, National Aeronautics and Space Administration.

[27] USDD (2006) Risk Management Guide for DoD Acquisition. United States Department of Defense.

[28] Belloví, M.B. and Malagón, F.P. (1993) Sistema simplificado de evaluación de riesgos de accidente. Nota Técnica de Prevención 330, Instituto Nacional de Seguridad y Higiene en el Trabajo, Barcelona.

[29] Cox, L.A.J. (2008) What's Wrong with Risk Matrices? Risk Analysis, 28, 497-512. https://doi.org/10.1111/j.1539-6924.2008.01030.x

[30] BS 8800 (2004) Guide to Occupational Health and Safety Management Systems. Health and Safety Commission, British Standard Institution.

[31] Pasman, H.J., Jung, S., Prem, K., Rogers, W.J. and Yang, X. (2009) Is Risk Analysis a Useful Tool for Improving Process Safety? Journal of Loss Prevention in the Process Industries, 22, 769-777. https://doi.org/10.1016/j.jlp.2009.08.001

[32] Cox, L.A.J., Babayev, D. and Huber, W. (2005) Some Limitations of Qualitative Risk Rating Systems. Risk Analysis, 25, 651-662. https://doi.org/10.1111/j.1539-6924.2005.00615.x

[33] Malchaire, J. and Koob, J.-P. (2006) Fiabilité de la méthode Kinney d'Analyse des Risques. Médicine du Travail et Ergonomie, 43, 3-8.

[34] Kaplan, S. and Garrick, B.J. (1981) On the Quantitative Definition of Risk. Risk Analysis, 1, 11-27. https://doi.org/10.1111/j.1539-6924.1981.tb01350.x

[35] Hollnagel, E. (1999) Accident Analysis and Barrier Functions. Project TRAIN, Institute for Energy Technology, Kjeller, Norway.

[36] Sklet, S. (2006) Safety Barriers: Definition, Classification, and Performance. Journal of Loss Prevention in the Process Industries, 19, 494-506. https://doi.org/10.1016/j.jlp.2005.12.004

[37] Harms-Ringdahl, L. (2009) Analysis of Safety Functions and Barriers in Accidents. Safety Science, 47, 353-363. https://doi.org/10.1016/j.ssci.2008.06.004

[38] Chen, Q., Wu, W. and Zhang, X. (2012) The Differentiation and Decision Matrix Risk Assessment of Accident Precursors and Near-Misses on Construction Sites. International Journal of Engineering \& Technology IJET-IJENS, 12, 38-53.

[39] Cuny, X. and Lejeune, M. (1999) Occupational Risks and the Value and Modeling of a Measurement of Severity. Safety Science, 31, 213-229. https://doi.org/10.1016/S0925-7535(98)00067-8

[40] Cuny, X. and Lejeune, M. (2003) Statistical Modeling and Risk Assessment. Safety Science, 41, 29-51. https://doi.org/10.1016/S0925-7535(01)00056-X

[41] Gennarelli, A. and Wodzin, E. (2006) A Contemporary Injury Scale. Injury-International Journal of the Care of the Injured, 37, 1083-1091. https://doi.org/10.1016/j.injury.2006.07.009

[42] Papazoglou, I.A. and Ale, B.M. (2007) A Logical Model for Quantification of Occupational Risk. Reliability Engineering and System Safety, 92, 785-803. https://doi.org/10.1016/j.ress.2006.04.017

[43] Aneziris, O.N., Papazoglou, I.A., Baksteen, H., Mud, M., Ale, B.J., Bellamy, L.J., Hale, A.R., 
Bloemhoff, A., Post, J. and Oh, J.H. (2008) Quantified Risk Assessment for Fall from Height. Safety Science, 46, 198-220. https://doi.org/10.1016/j.ssci.2007.06.034

[44] Ale, B.M., Baksteen, H., Bellamy, L.J., Bloemhof, A., Goossens, L., Hale, A., Mud, M.L., Oh, J.H., Papazoglou, I.A., Post, J. and Whiston, J.Y. (2008) Quantifying Occupational Risk: The Development of an Occupational Risk Model. Safety Science, 46, 176-185. https://doi.org/10.1016/j.ssci.2007.02.001

[45] Snyder, R.G., Foust, D.R. and Bowman B.M. (1997) Study of Impact Tolerance through Free-Fall Investigations. Final Report, Highway Safety Research Institute, The University of Michigan, Ann Arbor.

[46] Cory, C.Z. and Jones, M.D. (2006) Development of a Simulation System for Performing in Situ Surface Tests to Assess the Potential Severity of Head Impacts from Alleged Childhood Short Falls. Forensic Science International, 163, 102-114. https://doi.org/10.1016/j.forsciint.2005.11.014

[47] Pinedo, I.F. (1982) Construcción de una escala de actitudes tipo Likert. Nota Técnica de Prevención 15, Instituto Nacional de Seguridad y Higiene en el Trabajo, Barcelona.

[48] ILO (1998) Resolution Concerning Statistics of Occupational Injuries (Resulting from Occupational Accidents). 16th International Conference of Labor Statisticians, International Labour Organization, Report III, 18-22.

[49] EU-OSHA (2011) Occupational Safety and Health Culture Assessment-A Review of Main Approaches and Selected Tools. European Agency for Safety and Health at Work.

[50] Gillen, M., Schneider, S., Hecker, S. and Goldenhar, L. (2013) Safety Culture and Climate in Construction: Bridging the Gap Between Research and Practice. Report from a Joint Workshop between the Center for Construction Research and Training (CPWR) and the National Institute for Occupational Safety and Health (NIOSH).

[51] Nunes, F.O. (2011) Information System for the Management of Occupational Health and Safety in the Organizations. PhD Thesis, Universidad de León, Leon.

Submit or recommend next manuscript to SCIRP and we will provide best service for you:

Accepting pre-submission inquiries through Email, Facebook, LinkedIn, Twitter, etc.

A wide selection of journals (inclusive of 9 subjects, more than 200 journals)

Providing 24-hour high-quality service

User-friendly online submission system

Fair and swift peer-review system

Efficient typesetting and proofreading procedure

Display of the result of downloads and visits, as well as the number of cited articles

Maximum dissemination of your research work

Submit your manuscript at: http://papersubmission.scirp.org/

Or contact ojsst@scirp.org 